\title{
Comment
}

\section{How OR/MS reflects cultural differences ${ }^{1}$}

\section{HEINER MÜLLER-MERBACH}

What are the differences between OR/MS in different countries, such as the UK and North America, Japan and Continental Europe, China and India, Australia and South Africa, Latin America and Southern Europe, etc.?

There are no differences for those who understand OR as a subset of mathematics. Linear programming, graphs, queues, simulation, branch and bound, dynamic programming, stochastic processes - all the models structures (in mathematical terms), the algorithms, the theorems and proofs are identical.

The differences are fundamental, however, for those who understand OR/MS as intervention into management processes, participation in problem-solving and decision-making, and interaction with leaders. The cultural structures are different, the means and ends differ, the problems have different properties and the decision processes vary from country to country. Hence, the conditions for OR/MS differ remarkably (even if the mathematical tools remain the same), and OR/MS as such has quite a different meaning and plays quite a different role in different countries. In fact, it is one of the purposes of IFORS to make the differences understood and to maintain the international exchange in order to understand such differences.

\section{Why are the conditions different?}

The sources of wealth are different. Some nations have a mainly agricultural production structure, others depend upon their rich natural resources, others upon their industrial excellence, ingenuity and efficiency, others upon their international banking and services. Hence, the demand for OR/MS advice varies.

The management processes and leadership cultures are different. The negotiation attitudes may be based on rational analysis of facts and figures, or they may be driven by beliefs in harmony and consensus, or they may be more emotional. In any case, intervention, participation and interaction of OR/MS has to be adapted to the management and leadership style.

Different systems of co-determination and industrial democracy are being practised.

The systems of education are quite different, including those of higher education. Some countries offer one degree university courses (i.e. the Diploma) while others offer two degree courses (i.e. Bachelor and Master). There are differences in the disciplines too. Some countries provide degrees in statistics, others do not; some provide degrees in industrial engineering and others do not; in some countries business administration has a rich tradition, but in others it has not. Due to these differences one may find OR/MS education in close neighbourhood to statisics, industrial engineering, production engineering, business administration, mathematics, or computer sciences, etc., respectively. As a consequence, the OR/MS expertise finds quite different channels to industrial and administrative practice.

OR/MS is in a similar position to many other new fields of scientific endeavour which have the potential of providing management and decision support, i.e. cybernetics, business informatics, artificial intelligence, expert systems, etc. As long as a merely technical understanding of such fields is prevailing, it can be anticipated that their practical influence will be quite limited. But as soon as the communities of such disciplines understand their roles as intervention, participation and interaction, these disciplines will have quite an impact on this world. However, this requires the adaptation of the disciplines to their national cultural structures.

${ }^{1}$ IFORS letter from the President No.28, April 1988.

Professor HEINER MÜLLER-MERBACH is a former President of the International Federation of Operational Research Societies. He was recently elected a Companion of OR by the OR Society. 